\title{
Interleukin-10 gene transfer into insulin-producing $\beta$ cells protects against diabetes in non-obese diabetic mice
}

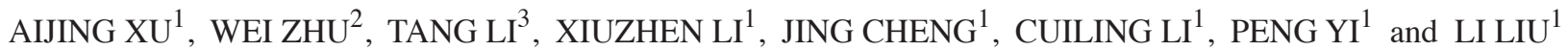 \\ ${ }^{1}$ Department of Pediatric Endocrinology and Metabolism, \\ Guangzhou Women and Children's Medical Center, Guangzhou Medical College, Guangzhou, Guangdong 510520; \\ ${ }^{2}$ Guangdong Provincial Key Laboratory of Medical Molecular Diagnostics, Guangdong Medical College, Dongguan, \\ Guangdong 523808; ${ }^{3}$ Department of Pediatrics, The Affiliated Hospital of Qingdao Medical College, \\ Qingdao University, Qingdao, Shandong 266003, P.R. China
}

Received May 16, 2014; Accepted March 4, 2015

DOI: $10.3892 / \mathrm{mmr} .2015 .3809$

\begin{abstract}
Type 1 diabetes is an autoimmune disorder, which occurs due to $\beta$ cell damage. Interleukin (IL)-10, a pleotropic cytokine, has been reported to have anti-inflammatory, immunosuppressive and immunostimulatory properties. Administration of IL-10 is known to prevent autoimmune diabetes in non-obese diabetic (NOD) mice. However, the mechanism of IL-10-induced protection in NOD mice requires further investigation. The aim of the present study was to evaluate the protective effect of transgenic IL-10 expression in pancreatic $\beta$ cells against autoimmune damage in NOD mice and to elucidate its mechanism of action. Female NOD mice ( 9 weeks old) were intraperitoneally injected with an adenovirus carrying either IL-10 (Adv-IL-10) or green fluorescent protein (Adv-GFP). Blood glucose was monitored weekly and the expression of IL-10 was evaluated using reverse transcription quantitative polymerase chain reaction. IL-10 and interferon (IFN)- $\gamma$ expression levels in serum and splenocytes were analyzed. $\mathrm{CD} 4{ }^{+} \mathrm{CD} 25^{+} \mathrm{FoxP} 3^{+} \mathrm{T}$ regulatory (Treg) cells were determined by flow cytometry. Apoptosis of pancreatic $\beta$ cells was determined using a terminal deoxynucleotidyl transferase deoxyuridine triphosphate nick-end labeling assay and expression levels of Fas and caspase-3 were estimated by immunohistochemistry analysis. The results revealed that mice treated with IL-10 showed less severe insulitis and a lower
\end{abstract}

Correspondence to: Professor Wei Zhu, Guangdong Provincial Key Laboratory of Medical Molecular Diagnostics, Guangdong Medical College, 1 Park Avenue, Dongguan, Guangdong 523808, P.R. China

E-mail: zhuwei168@yahoo.cn

Professor Li Liu, Department of Pediatric Endocrinology and Metabolism, Guangzhou Women and Children's Medical Center, Guangzhou Medical College, 9 Jinsui Road, Guangzhou, Guangdong 510520, P.R. China

E-mail: liliuxia@hotmail.com

Key words: interlukin-10, non-obese diabetic mice, apoptosis, caspase incidence of diabetes compared with the saline control and Adv-GFP groups. In addition, compared with the control group, IFN- $\gamma$ levels were decreased in sera and splenocytes, while IL-10 expression was increased in sera only. The number of $\mathrm{CD} 4{ }^{+} \mathrm{CD} 25^{+} \mathrm{FoxP}^{+}$Treg cells was increased in IL-10-injected mice. Furthermore, the expression levels of Fas and caspase-3 were decreased in IL-10-injected mice compared with that of GFP-injected and control mice, which was concomitant with a reduction in $\beta$ cell apoptosis. In conclusion, the present study demonstrated that IL-10 gene transfer reduced the expression of the inflammatory cytokines, attenuated pancreatic insulitis and inhibited $\beta$ cell apoptosis. This therefore indicated that IL-10 reduced the incidence of diabetes in female NOD mice.

\section{Introduction}

Type 1 diabetes mellitus (T1D) is an autoimmune disease that occurs due to autoreactive $\mathrm{T}$ cell-induced damage to pancreatic insulin-producing $\beta$ cells. In addition, it was reported that by the time the first clinical symptoms of T1D become evident, only $15-20 \%$ of $\beta$ cells remain $(1,2)$. Inflammatory cells in the islet milieu produce cytokines and cytokine-induced proteins, which have a critical role in the pathogenesis of insulitis and T1D. The onset of T1D is typically associated with altered cytokine expression, from primarily $\mathrm{T}$ helper cell (Th)2 to Th1 cytokines, which was thought to occur due to continuous inflammation in the pancreas (3). Therefore, regulating the immune system with regard to Th2-like immunity represents a potential therapeutic strategy for T1D (4). In animal models of T1D, approaches favoring Th2 activity have led to diabetes prevention; in addition, there is evidence to suggest that the administration of these immunoregulatory cytokines reduces autoimmune diabetes (4-6). The Th2-like cytokine interleukin (IL)-10 has been extensively investigated and was suggested to be a potential candidate for effective immune diversion for diabetes treatment (7).

IL-10 is known to be an anti-inflammatory cytokine, which exhibits potent suppressive effects in preventing autoimmune disease (8). IL-10 was reported to be effective in the suppression of cytokine production in T cells (IL-2), natural killer cells [interferon (IFN)- $\gamma$ ] and monocyte/macrophages [IL-1 $\beta$, 
IL-6, IL-8, IL-12, tumor necrosis factor (TNF)- $\alpha$ and granulocyte-macrophage colony-stimulating factor (GM-CSF)]; IL-10 was also implicated in the induction of anergy in T cells $(9,10)$. It was demonstrated that IL-10 overexpression prevented graft rejection in transplant organs (7). IL-10 treatment was shown to inhibit the onset of T1D in non-obese diabetic (NOD) mice and prevent disease recurrence, which was dependent on the time and mode of administration (early vs. late, systemic vs. local). Of note, systemic delivery of the IL-10 gene and IL-10 protein or virus-mediated IL-10 delivery was suggested to delay the onset of diabetes symptoms as well as reduce the occurrence of diabetes in NOD mice $(11,12)$.

IL-10 has been reported to serve as a growth factor for $\mathrm{T}$ regulatory cells (Tregs) $(13,14)$; it was demonstrated that IL-10 increased the $\mathrm{CD} 4{ }^{+} \mathrm{CD} 25^{+}$cell population in vitro and in vivo $(15,16)$. In addition, a previous study revealed that IL-10 gene transfer prevented IL-1 $\beta$-induced nitric oxide production in $\beta$ cells in vitro, which were also found to retain their insulin secretion function, even at high glucose levels (17). Furthermore, IL-10 overexpression inhibited IL-1 $\beta$-induced Fas expression and insulin secreting cell apoptosis (18). These findings supported the functional importance of IL-10 in immune regulation. In addition, evidence suggested that IL-10 exhibited a partial, direct protective effect on human islets exposed to pro-inflammatory and Th1 cytokines (19). However, to the best of our knowledge, few direct measurements have been published regarding the anti-apoptotic mechanism of IL-10 in vivo. Recombinant IL-10 protein treatment requires repeated or continuous administrations due to its short half-life. Therefore, viral vectors are a promising type of gene transfer vehicle, as they often mediate highly efficient gene transfer and stably express genes.

The aim of the present study was to assess the recombinant adenovirus-based transfer of genes encoding IL-10 to manipulate $\beta$ cell autoimmunity in NOD mice. The present study aimed to examine if overexpression of IL-10 in NOD mice was able to prevent the development of diabetes by downregulating experimental autoimmune disorders.

\section{Materials and methods}

Animals. A total of 42 ( $n=14 /$ group) female NOD mice, 8 weeks of age, were purchased from the Shanghai Laboratory Animal Center, Chinese Academy of Sciences (Shanghai, China) and housed in specific pathogen-free conditions. The animals were housed four per cage in a room, which was maintained under standard laboratory conditions $(12 \mathrm{~h}$ light/dark cycle), at a constant temperature $\left(25 \pm 1^{\circ} \mathrm{C}\right)$ and a humidity of $50-60 \%$. Adenovirus treatments of NOD mice were initiated at age 9 weeks, which was 2 weeks prior to diabetes onset (from age 11-24 weeks). Diabetes was defined as two consecutive blood glucose values $>250 \mathrm{mg} / \mathrm{dl}$ in NOD mice. A total of seven NOD mice were sacrificed by cervical dislocation at 12 weeks of age and blood samples (1 $\mathrm{ml})$ were obtained from the abdominal vein and stored at $-80^{\circ} \mathrm{C}$, until further use. The remainder of the mice were allowed to develop diabetes and were sacrificed 1 week post diabetes onset or at 30 weeks. All procedures were approved by the Ethics Committee for the Use of Experimental Animals at Guangzhou Women and Children's Medical
Center (Guangzhou, China). All efforts were made to minimize the animals' suffering.

Preparation of the recombinant adenovirus. An E1- and E3-deleted, first-generation recombinant adenovirus (replication-deficient) was used as a vector in these experiments. The recombinant Adv-IL-10 vector was generated at the Qingdao University (Qingdao, China) by triple transfection protocol as previously described (18). Briefly, the IL-10 gene was cloned into the shuttle vector, pAdTrack-CMV, and the produced plasmid was linearized with PmeI digestion (New England Biolabs, Beverly, MA, USA). The linearized plasmid was co-transfected into Escherichia coli BJ5183 cells with an adenoviral backbone plasmid, pAdEasy-1. The recombinant adenovirus encoding murine IL-10 (Adv-IL-10) was confirmed by restriction endonuclease analysis. The linearized recombinant plasmid was transfected into HEK293 cells (kindly provided by Professor Bing Luo, Medical College of Qingdao University, China) and high-titer viral stocks were prepared. The adenovirus vector expressing enhanced green fluorescent protein was also produced for use as the viral control. Viruses were then purified from HEK293 cells by freeze/thaw cycles and cesium chloride (Solarbio Science \& Technology. Co., Ltd., Beijing, China) step gradients. All the viral titers were $5.5 \times 10^{10}$ plaque forming unit $/ \mathrm{ml}$. Aliquots of the obtained recombinant adenoviruses were frozen and stored in the ultra-low temperature freezers (Thermo Forma 900; Thermo Fisher Scientific, Inc., MA, USA).

Treatment of NOD mice with Adenoviruses. Female NOD mice were intraperitoneally (IP) injected with $100 \mu \mathrm{l} \mathrm{Adv-IL-10} \mathrm{or}$ Adv-GFP $\left(1 \times 10^{10} \mathrm{pfuv} / \mathrm{ml}\right)$. Age-matched mice were injected with $100 \mu \mathrm{l}$ normal saline (Hualu Pharmaceutical Co., Ltd, Shandong, China) as controls. The general condition (i.e. alertness and physical activity) and body weight of mice were observed and measured weekly.

Incidence rate of T1D. During the experimental period, blood samples $(0.5 \mathrm{ml})$ were taken weekly from the tail vein of NOD mice and glucose levels were measured using a one-touch ultra glucometer (Johnson \& Johnson Medical Ltd, Suzhou, China). When glucose levels exceeded $250 \mathrm{mg} / \mathrm{dl}$ on two consecutive occasions (initial measurement and then measured again $24 \mathrm{~h}$ later), animals were considered to have T1D and were sacrificed 1 week post diagnosis for pathological examinations.

Reverse transcription polymerase chain reaction (RT-PCR) for detecting transgene expression. Prior to sacrifice, splenocytes and liver were isolated from all NOD mice. The spleens were harvested and pressed through a nylon mesh to produce a single-cell suspension. The red blood cells were treated with ACK lysis buffer (Beyotime Institute of Biotechnology, Shanghai, China) for $5 \mathrm{~min}$. The isolated splenocytes were washed three times and resuspended in RPMI-1640 (Sigma-Aldrich, St. Louis, MO, USA), supplemented with $10 \%$ fetal calf serum (FCS; Gibco Life Technologies, Carlsbad, CA, USA), penicillin and streptomycin (Gibco Life Technologies) for the subsequent experiments. Total RNA ( $1 \mu \mathrm{g}$ from liver and spleen) was extracted and purified using TRIzol ${ }^{\circledR}$ (GE Healthcare, Shanghai, China) according 
to the manufacturer's instructions. Equal amounts $(1 \mu \mathrm{g})$ of RNA were then reverse-transcribed into complementary DNA using a Prime Script RT Reagent kit (Takara Bio, Inc., Dalian, China). The primers (Sangon Biotech Co., Ltd., Shanghai, China) used for PCR were as follows: IL-10 forward, 5'-GGCATGCTTGGCTCAGCACTG-3' and reverse, 5'-GCCCTGCAGTCCAGTAGACG-3' (580 bp). Cycling conditions were as follows: Initial activation at $95^{\circ} \mathrm{C}$ for $5 \mathrm{~min}$, followed by 40 cycles of $95^{\circ} \mathrm{C}$ for $30 \mathrm{sec}, 55^{\circ} \mathrm{C}$ for $60 \mathrm{sec}$ and $72^{\circ} \mathrm{C}$ for $60 \mathrm{sec}$. The RT-PCR products were detected using $1.2 \%$ agarose gel electrophoresis (Qiagen, Hilden, Germany). The products were visualized by staining with ethidium bromide (Solarbio Biotech Co., Ltd.), observed and photographed using Bio-Rad gel imaging system (Bio-Rad Gel Doc 2000 UV Chemi Doc; Bio-Rad Laboratories, Inc., Pleasanton, CA, USA).

Histological and morphological analyses. Pancreatic tissues from Adv-IL-10- and Adv-GFP-treated NOD mice were removed, fixed in $10 \%$ buffered formalin (Xilong Chemical Co., Ltd., Guangzhou, China), embedded in paraffin (Junjie Electronics Co., Ltd., Wuhan, China) and sectioned at 5- $\mu \mathrm{m}$ thickness. Sections were stained with hematoxylin and eosin (Beyotime Institute of Biotechnology) to assess pancreatic islets histology and morphology in the experimental animals. Between 5 and 10 islets of each pancreatic tissue sample were observed and the inflammation of the islets was graded. The grading of insulitis was scored as follows: 0 , no inflammation; 1 , peri-insulitis; 2 , inflammatory infiltration $<50 \%$ of islet area; and 3, inflammation $>50 \%$ of islet area and islet structure disruption (20).

Analyses of serum cytokine levels. Sera from Adv-IL-10- or Adv-GFP-treated NOD mice were frozen at $-20^{\circ} \mathrm{C}$ and stored until further use. Quantitative determination of cytokine (IL-10 and IFN- $\gamma$ ) production was assessed using a murine IL-10 and a murine IFN- $\gamma$ ELISA commercial kits (R\&D System, Inc., Minneapolis, MN, USA). The optical density (OD) value was determined at $450 \mathrm{~nm}$ using an ELISA reader (Bio-Rad Laboratories, Inc.) at $405 \mathrm{~nm}$. The amount of cytokine present was determined from standard curves using purified recombinant cytokines.

Analyses of cytokine-secreting splenocytes. Splenocytes were collected and stimulated with $10 \mu \mathrm{g} / \mathrm{ml}$ Concanavalin A (Sigma-Aldrich, St. Louis, MO, USA). Following incubation for $48 \mathrm{~h}$, the culture supernatants were harvested for determination of IL-10 and IFN- $\gamma$ using ELISA kits (R\&D Systems, Inc.) according to the manufacturer's instructions. All experiments were performed in triplicate for each sample.

Flow cytometric analysis of Treg cells in IL-10-treated NOD mice. NOD mice were randomly selected for the detection of Tregs in the spleen. These mice were sacrificed following IL-10 treatment. Cells from the spleen were prepared and incubated with $1 \mu \mathrm{g}$ anti-mouse monocolnal CD4, anti-mouse monoclonal CD25 and anti-mouse monoclonal Foxp3 conjugated with fluorescein isothiocyanate, phycoerythrin (PE) or CyChrome, respectively (eBioscience, San Diego, CA, USA) on ice for $30 \mathrm{~min}$. Cells were then washed in phosphate-buffered saline (PBS; Solarbio Biotech Co., Ltd.)-2\% fetal calf serum (Gibco Life Technologies). Stained cells were analyzed using a FACSCalibur flow cytometer (Becton-Dickinson, San Jose, CA, USA).

Terminal deoxynucleotidyl transferase deoxyuridine triphosphate (dUTP) nick-end labeling (TUNEL) assay. TUNEL staining was performed in order to detect apoptosis using an in situ cell death detection kit (Roche, Indianapolis, IN, USA). Paraffin sections ( $4 \mu \mathrm{m}$ ) were deparaffinized and dehydrated in serial alcohol concentrations $(100,95,90,80$ and $70 \%$, diluted in double distilled water). Protein was digested using proteinase $\mathrm{K}$ (Sigma-Aldrich) at $37^{\circ} \mathrm{C}$ for $10 \mathrm{~min}$ and then incubated with terminal transferase and digoxigenin-dUTP. Endogenous peroxidase was quenched with $3 \% \mathrm{H}_{2} \mathrm{O}_{2}$ (Xilong Chemical Co., Ltd.) in methanol (Xilong Chemical Co., Ltd.) for $5 \mathrm{~min}$. Labeled end was detected using streptavidin-horseradish peroxidase conjugate. Specimens were further processed with 3,3-diaminobenzidine coloration (Sigma-Aldrich) and slightly redyed with Mayer hematoxylin (Sigma-Aldrich). Specimens were subsequently washed with PBS twice for 5 min each. The sections were susbequently incubated with $50 \mu 1$ TUNEL reaction mixture at $37^{\circ} \mathrm{C}$ for $1 \mathrm{~h}$ in a humidified chamber. Following incubation, the slides were rinsed with PBS three times for $5 \mathrm{~min}$ each and the apoptotic cells were visualized using an Olympus BX40F microscope (Olympus, Melville, NY, USA). Apoptotic cells were identified by their dark brown nuclei under a light microscope (Olympus IX81; Olympus, Tokyo, Japan). The number of apoptotic cells was counted in five randomly selected fields of vision (magnification, $x 400$ ) in a blinded manner.

Immunohistochemical analysis. Fas staining was performed in order to determine the presence of apoptosis pathway protein and caspase-3 staining was used to detect early apoptosis. Briefly, the pancreata were fixed overnight in $10 \%$ formalin and embedded in paraffin. Tissues were then sectioned (4 $\mu \mathrm{m}$-thick) and deparaffinized. The endogenous peroxidase activity was quenched by $3 \%$ hydrogen peroxidemethanol (Boster Biological Technology, Ltd., Wuhan, China) for $10 \mathrm{~min}$ at room temperature. Samples were then incubated overnight with primary antibodies against rabbit anti-caspase-3 polyclonal antibody and rabbit anti-Fas rabbit polyclonal antibody (1:100; BD Pharmingen, San Diego, CA, USA) at $4^{\circ} \mathrm{C}$. Following washing with PBS, samples were incubated with biotinylated secondary antibody (Boster Biological Technology, Ltd.) for $20 \mathrm{~min}$ at $37^{\circ} \mathrm{C}$. Slides were visualized with diaminobenzidine (Boster Biological Technology, Ltd, Wuhan, China), counterstained with hematoxylin, dehydrated through increasing concentrations of alcohol, cleared in xylene and then observed with microscope (Olympus). Images were captured using a Sony 3CCD color video camera (Sony, Tokyo, Japan).

Statistical analysis. Values are presented as the mean \pm standard error of the mean. Parametric tests or non-parametric tests were performed to determine statistically significant differences between groups, as appropriate, using SPSS 17.0 software (International Business Machines, Armonk, NY, USA). Student's t-test was used for statistical 


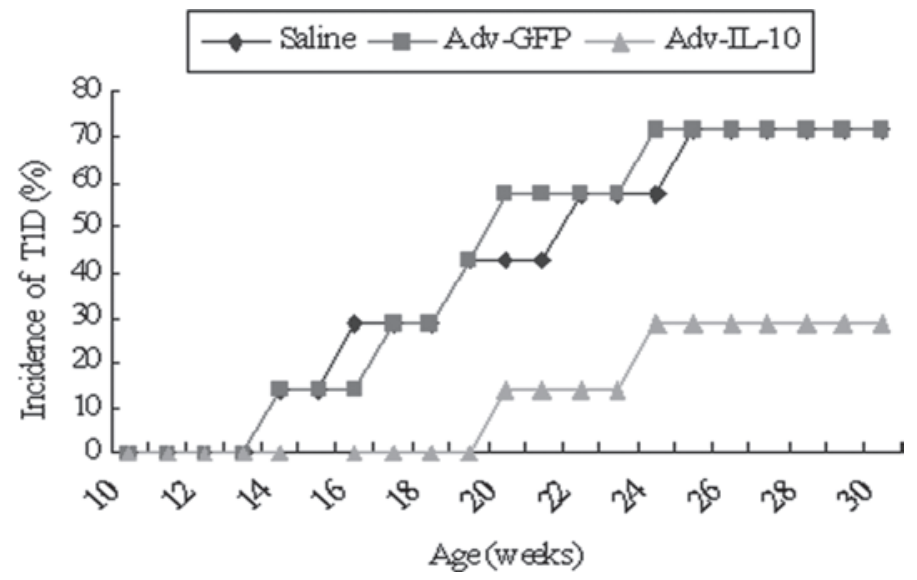

Figure 1. Cumulative incidence rate of T1D in NOD mice administered Adv-IL-10, Adv-GFP or saline. During the experimental period, blood samples were obtained from the tail veins of NOD mice weekly and glucose levels were measured using a glucometer. Diabetes diagnosis was defined by a blood glucose level $>250 \mathrm{mg} / \mathrm{dl}$ on two consecutive measurements, $>24 \mathrm{~h}$ apart. The incidence rate of T1D was reduced from $71.4 \%$ in the saline and Adv-GFP groups to 28.6\% (P<0.05). T1D, type 1 diabetes mellitus; NOD, non-obese diabetic; Adv-IL-10, adenovirus carrying interleukin-10; Adv-GFP, adenovirus carrying green fluorescent protein.
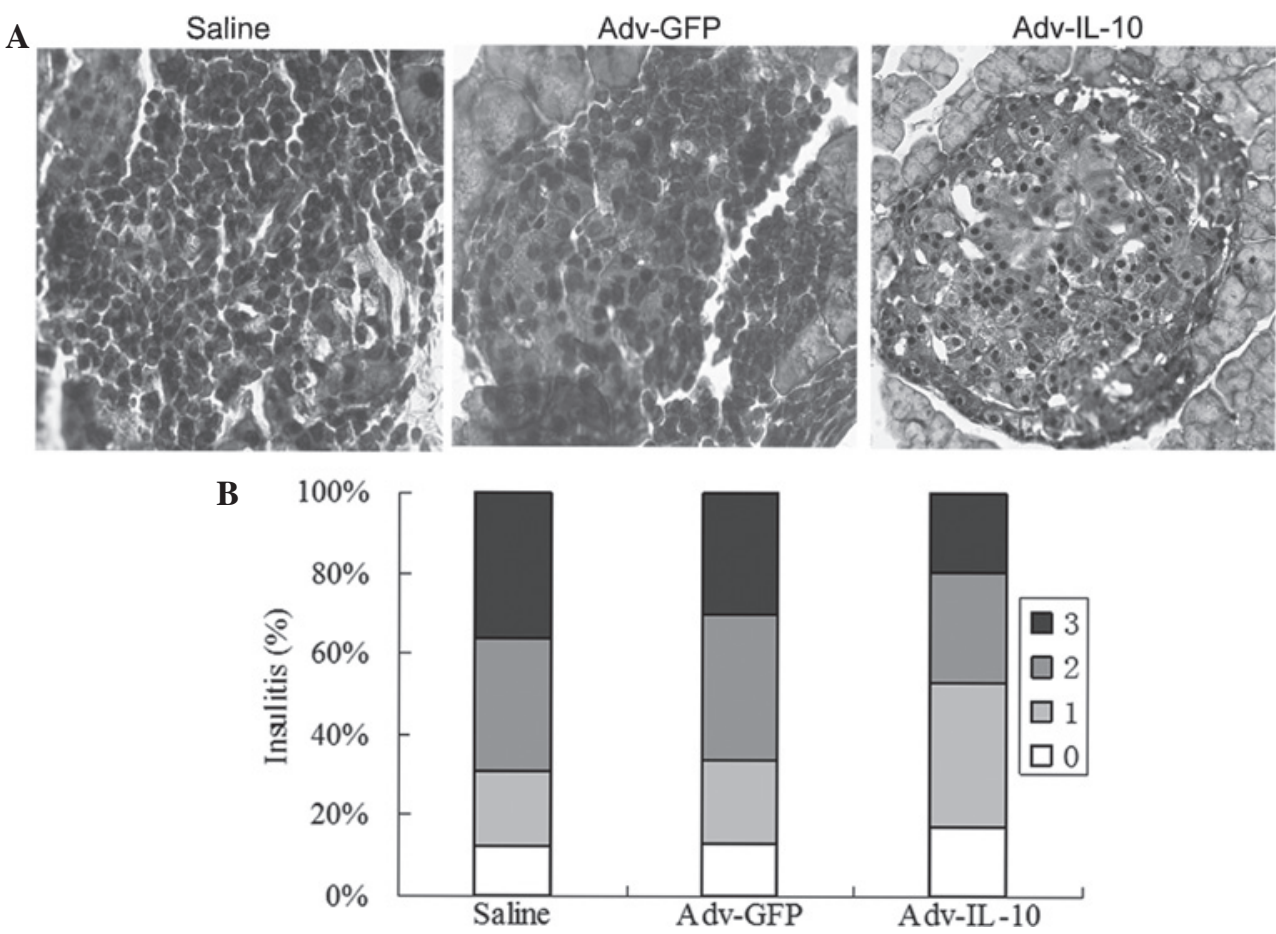

Figure 2. IL-10 gene transfer reduces insulitis. (A) Pancreatic sections were stained with hematoxylin and eosin, then assessed for insulitis. For each mouse, five random sections were scored ( $n=7$ per group; magnification, $x 400)$. A representative image from each group is shown. (B) Histograms depict the percentage of normal islets (stage 0 , unfilled bar), peri-insulitis (stage 1 , light gray bar), insulitis involving $<50 \%$ of the islet in cross-section (stage 2 , dark gray bar) or insulitis involving $>50 \%$ of the islet (stage 3, black bar). IL-10, interleukin-10; Adv-GFP, adenovirus carrying green fluorescent protein; Adv-IL-10, adenovirus carrying IL-10.

analysis of the data. $\mathrm{P}<0.05$ was considered to indicate a statistically significant difference between values.

\section{Results}

IL-10 gene transfer protects pancreatic $\beta$ cells from autoimmune attack in TID. To determine whether IL-10 gene overexpression delayed or prevented diabetes, 9-week-old NOD mice were IP injected with Adv-IL-10. As shown in Fig. 1, the incidence of diabetes in Adv-GFP-injected NOD mice was $57.1 \%$ at 20 weeks, which increased to $71.4 \%$ by 30 weeks of age. By contrast, only
$28.6 \%$ of Adv-IL-10-injected NOD mice developed diabetes throughout the entire experimental protocol (Fig. 1).

Gene transfer of IL-10 reduces insulitis in prediabetic NOD mice. It was investigated whether the insulitis of NOD mice was suppressed following IP injections of Adv-IL-10 plasmid complexes. As shown in Fig. 2A, severe mononuclear cell infiltration was observed in and around islets in control mice groups. By contrast, IL-10-injected mice displayed reduced mononuclear cell infiltration and insulitis was only observed at the periphery of islets (peri-insulitis). Average insulitis scores 


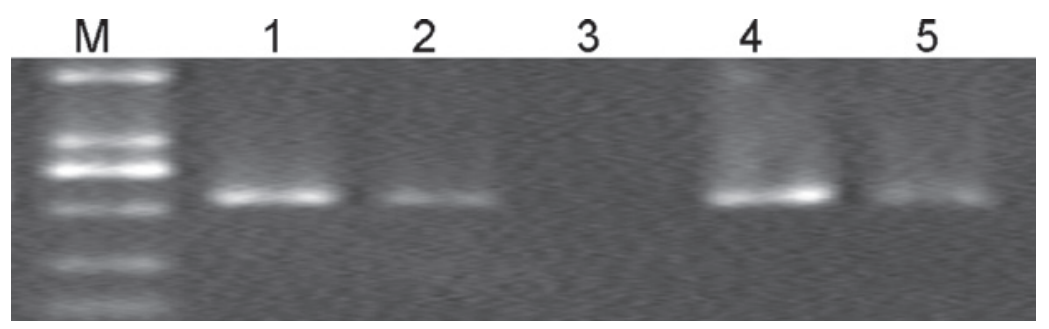

Figure 3. IL-10 messenger RNA expression in liver cells and splenocytes of non-obese diabetic mice, as determined by RT-PCR. Total RNA (5 $\mu \mathrm{g})$ of liver and splenocyte samples from non-obsese diabetic mice was used for RT-PCR. Lanes are representative results from each group as follows: M, DL2000 marker; 1 and 2, liver from mice injected with Adv-IL-10 or Adv-GFP, respectively; 3, saline-injected mice; 4 and 5, isolated splenocytes from mice injected with Adv-IL-10 or Adv-GFP, respectively. Experiments were performed in triplicate ( $\mathrm{n}=3$ per group). IL-10, interleukin-10; RT-PCR, reverse transcription polymerase chain reaction; Adv-GFP, adenovirus carrying green fluorescent protein; Adv-IL-10, adenovirus carrying IL-10.

A
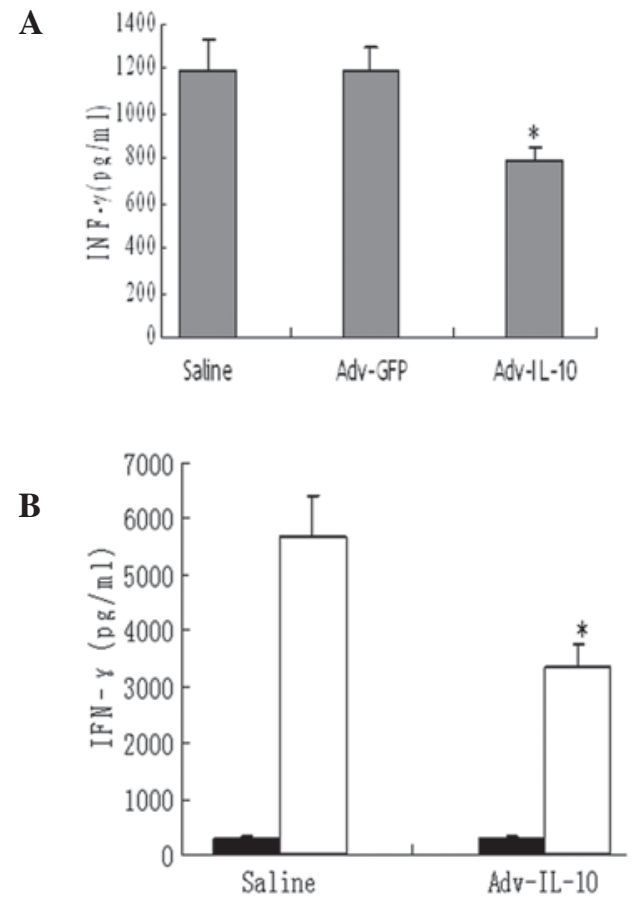
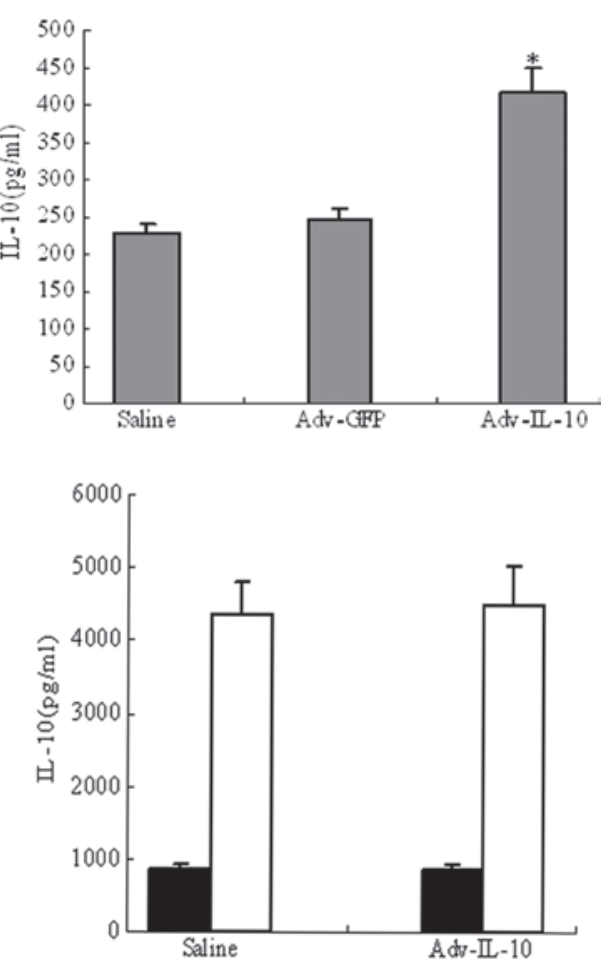

Figure 4. Cytokine protein expression levels in sera and splenocyte media of non-obese diabetic mice. (A) Serum INF- $\gamma$ and IL-10 levels in mice administered saline, Adv-GFP or Adv-IL-10. (B) INF- $\gamma$ and IL-10 levels in culture medium of splenocytes from saline- and Adv-IL-10-treated groups in the absence (black) or presence (white) of Concanavalin A activation. ${ }^{*} \mathrm{P}<0.05$ vs. saline-treated group. INF- $\gamma$, interferon- $\gamma$; IL-10, interleukin-10; Adv-GFP, adenovirus carrying green fluorescent protein; Adv-IL-10, adenovirus carrying IL-10.

for mice receiving the IL-10 virus were decreased compared with those of the control mice groups (Fig. 2B). The transfer of the IL-10 gene into islets can lessen the degree of lymphocytic infiltration. These results indicated that systemic overexpression of IL-10 protected pancreatic islets against immunological destruction.

RT-PCR detection of IL-10 messenger (m)RNA in NOD mice. To evaluate whether transgene expression of IL-10 was involved in the development of autoimmune diabetes, a RT-PCR assay of total RNA prepared from the liver and splenocytes of NOD mice was developed to detect transcripts of IL-10 genes. The results showed that mRNA levels of IL-10 in the liver and splenocytes were increased in the Adv-IL-10-treated mice compared with those of mice in the control groups (Fig. 3). This demonstrated that Adv-IL-10 expressed IL-10 genes with high efficiency.
Effect of Adv-IL-10 delivered immunomodulatory cytokines on T1D. In order to determine whether the balance of Th1/Th2 responses was affected by IL-10 expression, Th1 and Th2 cytokine levels were determined in serum samples and in media from splenocytes isolated from animals 3 weeks following saline or Adv-IL-10 administration. As shown in Fig. 4A, serum levels of IFN- $\gamma$ were significantly decreased in mice injected with Adv-IL-10 compared with the saline and Adv-GFP control groups $(\mathrm{P}<0.05)$; however, serum levels of IL-10 were increased in the Adv-IL-10-treated group $(\mathrm{P}<0.05)$. In addition, the production of IFN- $\gamma$ by activated splenocytes was substantially lower in Adv-IL-10-treated mice $(\mathrm{P}<0.05)$; however, IL-10 levels were not significantly different between the saline- and Adv-IL-10-treated groups (Fig. 4B).

Treg cell activity in vivo. Previous studies have highlighted the role of IL-10 in the function of Treg cells $(15,16)$. The 
A

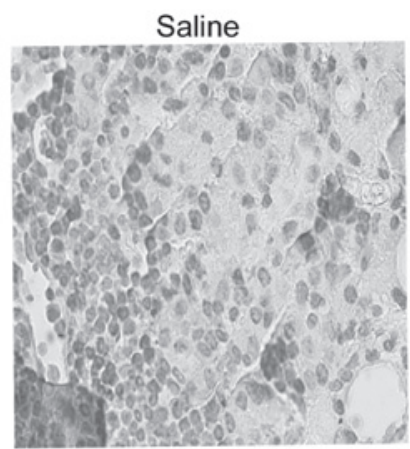

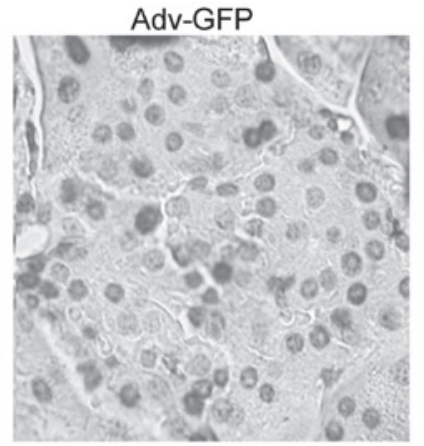

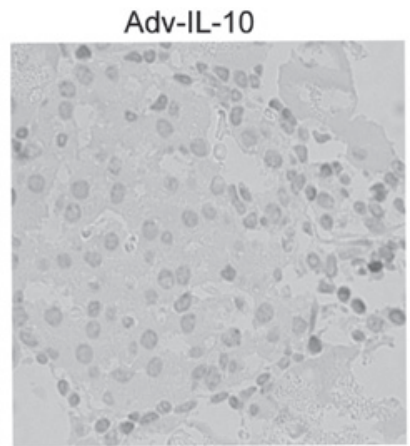

B

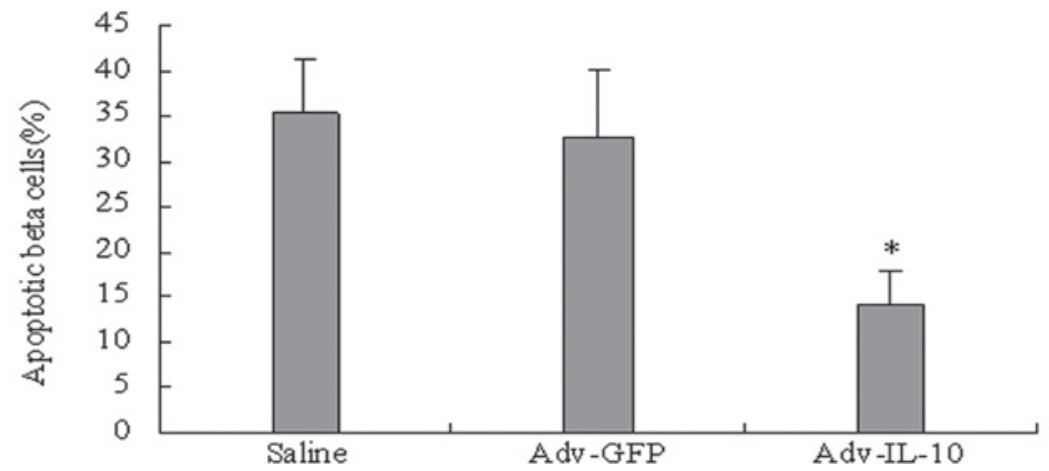

Figure 5. IL-10 overexpression protects islets from $\beta$ cell apoptosis. (A) Representative images of terminal deoxynucleotidyl transferase deoxyuridine triphosphate nick-end labeling assay for apoptotic $\beta$ cells following treatment with saline, Adv-GFP or Adv-IL-10 (magnification, x400). (B) Percentage of apoptotic $\beta$ cells was determined in each group. Values are presented as the mean \pm standard error of the mean of five randomly selected fields of vision ( $n=7$ per group). IL-10, interleukin-10; Adv-GFP, adenovirus carrying green fluorescent protein; Adv-IL-10, adenovirus carrying IL-10.

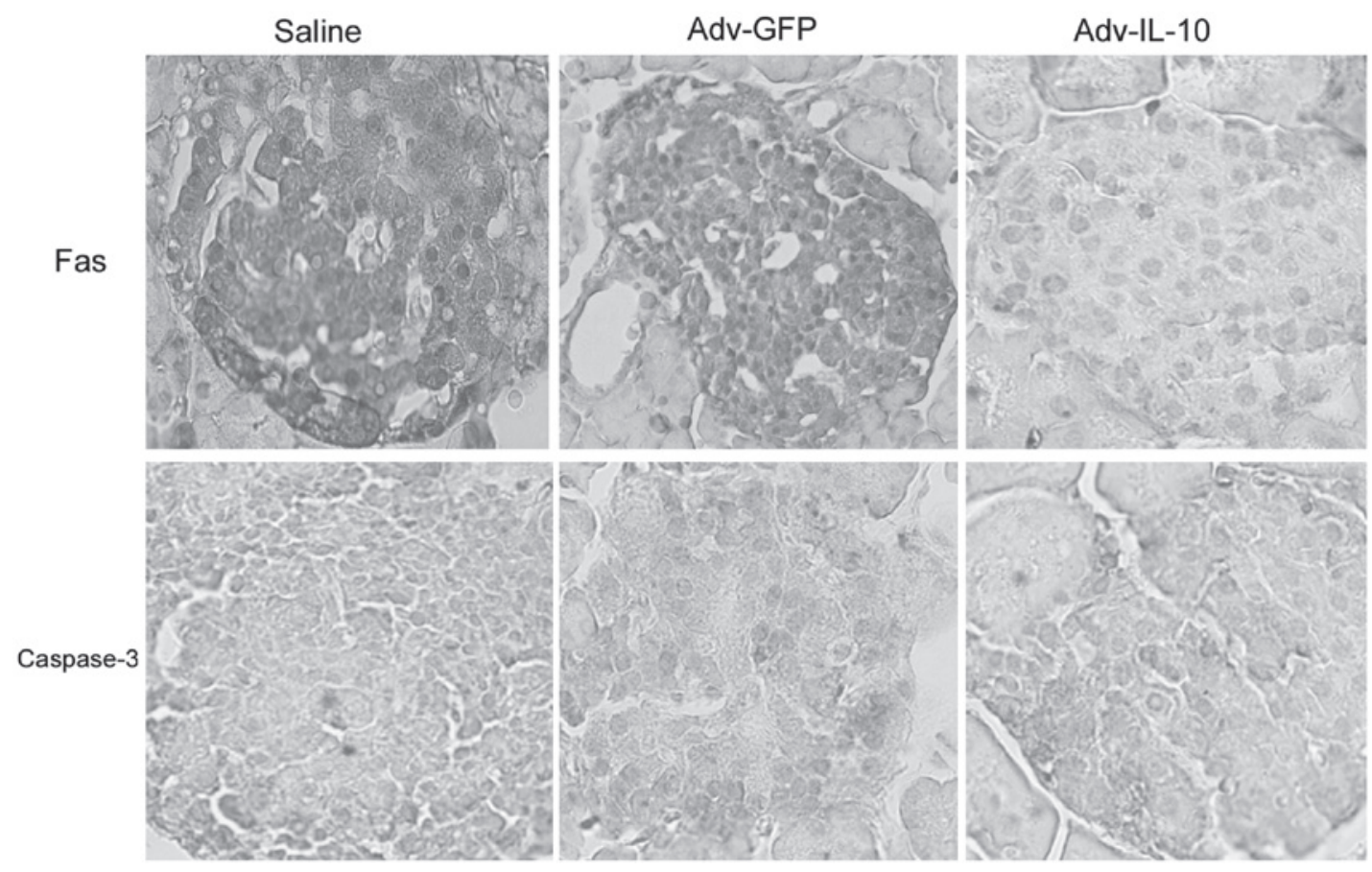

Figure 6. Immunohistochemical detection of Fas and caspase-3 protein expression in pancreatic islets from saline-, Adv-GFP- and Adv-IL-10-treated mice (magnification, x400). Adv-IL-10, adenovirus carrying interleukin-10; Adv-GFP, adenovirus carrying green fluorescent protein.

present study investigated the effect of IL-10 gene therapy on the function of Tregs. NOD mice were treated with Adv-IL-10 or saline and flow cytometry was then used to investigate the presence of Tregs in pancreatic islets. Flow cytometric analysis showed that the number of $\mathrm{CD} 4^{+} \mathrm{CD} 25^{+} \mathrm{Foxp} 3^{+}$Treg cells in the Adv-IL-10 group (7.03 $\pm 0.30 \%)$ was signifi- cantly increased compared with the saline $(6.63 \pm 0.44 \%)$ or Adv-GFP (6.58 $\pm 0.53 \%)$ controls $(\mathrm{P}<0.01 ; \mathrm{n}=7)$.

IL-10 therapies prevent $\beta$ cell apoptosis. As shown in Fig. 5 , the apoptotic rate of $\beta$ cells was decreased in mice treated with IL-10. The apoptosis rate of $\beta$ cells was lower in the Adv-IL-I0 
group (14.10 $\pm 2.70 \%)$ compared with the Adv-GFP group $(32.80 \pm 7.5 \%)$ and the normal control group $(35.40 \pm 6.10 \%$; $\mathrm{P}<0.05$ ) by TUNEL assay (Fig. 5A and B). Consistent with the results of the TUNEL staining assay, staining for caspase-3 showed a significant reduction in caspase-3 expression in IL-10-treated mice $(28.6+4.64 \% ; n=7)$ compared with the control $(48.8+10 \%)$ and Ad-GFP $(42.5 \pm 7.8 \%)$ groups These data indicated that IL-10 blocked general apoptotic pathways via inhibition of caspase-3. Therefore, the present study examined whether the decreased apoptosis observed in the islets of mice treated with Adv-IL-10 were associated with the downregulation of the anti-apoptotic protein Fas (Fig. 6). Immunohistochemical staining revealed that Fas and caspase-3 protein expression were markedly reduced in the pancreatic islets of mice treated with the Adv-IL-10 compared with mice in the Adv-GFP- and saline-treated groups.

\section{Discussion}

T1D occurs due to cell-mediated autoimmune-associated damage of pancreatic insulin-producing $\beta$ cells (21). Pro-inflammatory cytokine-mediated pancreatic $\beta$ cell disruption is a critical pathological event in the pathogenesis of T1D (22). It was reported that Th1 cells were involved in establishing autoimmunity in NOD mice; in addition, endogenous Th2 cells were suggested to have a protective effect on the development of diabetes in mice (23). IL-10 exhibited pleiotropic effects in immunoregulation and inflammation (24); in addition, IL-10 was reported to have various roles in mediating autoimmune responses, which were dependent on the dosage and site of administration. For example, systemic administration of IL-10 was found to restrict the progression of insulitis and prevent the onset of diabetes (25). In NOD mice, systemic administration of recombinant adeno-associated virus (rAAV)- or plasmid-expressed IL-10 resulted in the prevention of spontaneous diabetes $(11,26)$. A previous study suggested that IFN- $\gamma / \mathrm{TNF}-\alpha$ synergism may be the final effector molecules involved in autoimmune diabetes in NOD mice (27). Serreze et al (23) reported that non-specific immunostimulatory agents prevented the development of diabetes in NOD mice, which was reported not to be dependent on the expression of the Th1 cytokine IFN- $\gamma$, rather than a Th1 to Th2 cytokine shift (23).

The present study aimed to examine the regulatory mechanisms of IL-10-induced protection against diabetes in NOD mice and it was observed that the injection of Adv-IL-10 into prediabetic NOD mice was able to significantly attenuate insulitis progression and the development of diabetes. RT-PCR revealed that IL-10 was highly expressed in islets of prediabetic NOD mice. In addition, the present study provided evidence to suggest that systemic IL-10 gene transfer suppressed the Th1 cell response in NOD mice islets as wells as significantly reduced the expression of Th1-type cytokine IFN- $\gamma$ in sera and in activated splenocytes. These results suggested that inhibition of IFN- $\gamma$ expression by IL-10 may, at least in part, contribute to the anti-inflammatory properties of IL-10 in islet inflammation.

Numerous previous studies have reported that Tregs have a critical role in the maintenance of peripheral pancreatic self-antigen tolerance (28-33). The decreased ability of Tregs to suppress autoreactive $\mathrm{T}$ cells was indicated to be associated with disease progression in mice and humans (34). Injection of polyclonal $\mathrm{CD}^{+}{ }^{+} \mathrm{CD} 25^{+} \mathrm{Foxp} 3^{+}$Treg cells into NOD mice was reported to prevent diabetes (35). Previous studies have indicated that systemic IL-10 overexpression in NOD mice enhanced the $\mathrm{CD} 4{ }^{+} \mathrm{CD} 25^{+}$Treg population and attenuated diabetes development (16). In addition, rAAV-IL-10 treatment was demonstrated to have a positive dose-dependent effect on the number of regulatory cells in vivo (16). The results of the present study demonstrated that the Adv-IL-10-treated group had an increased number of $\mathrm{CD} 4{ }^{+} \mathrm{CD} 25^{+}$Treg cells in the splenocyte population compared with that of the saline- and Adv-GFP-treated control mice. This therefore indicated that $\beta$ cell-specific IL-10 expression reduced the occurrence of disease-inducing lymphocytes, at least in part, through maintaining the number of Treg cells in pancreatic islets. It was therefore hypothesized that IL-10 treatment may also be involved in regulating the immune response, resulting in the prevention of diabetes.

Apoptosis is the primary mechanism of $\beta$ cell death in NOD mice (36). It was demonstrated that $\beta$ cell apoptosis induced a gradual decrease in the number of $\beta$ cells in rodent models of T1D (37). Autoreactive T lymphocytes are essential effector cells in murine autoimmune diabetes (38), which results in the induction of $\beta$ islet cells apoptosis $(39,40)$. Pro-inflammatory cytokines promote cell death via complex signaling pathways $(41,42)$, which result in the direct activation of caspases, the primary effector molecules of programmed cell death. The synergistic effect of IFN $\gamma$ and TNF $\alpha$ was reported to induce classical caspase-dependent apoptosis in murine insulinoma and pancreatic islet cells $(27,40)$; however, independently these cytokines exhibited no significant effects. The process of apoptosis involves caspase activity. Caspase-3 activation induces apoptosis in all cells previously examined, including $\beta$ cells, and is an early mediator of Fas signaling $(42,43)$. Fas (CD95) is a member of the TNF receptor superfamily. Normal human pancreatic $\beta$ cells do not constitutively express Fas; however, numerous studies on isolated rodent and human islets have demonstrated cytokine-induced Fas upregulation in the $\beta$ cells $(44,45)$. IL-10 has been reported to inhibit apoptotic pathways in different cell types (46-48), although there have been few studies regarding the protective effect of IL-10 against pro-inflammatory cytokine-induced $\beta$ cell apoptosis. The present study demonstrated that the apoptosis of $\beta$ cells was decreased in islets of Adv-IL-10-treated mice, which indicated that IL-10 production protected $\beta$ cells from death. This was consistent with previous studies that reported the direct protective effect of a combination of Th2 cytokines on human islet cells (19), as well as the anti-apoptotic effect of IL-10 in human promyeloid cells (46), cardiomyocytes (47) and endothelial cells (48). In addition, the present study demonstrated that IL-10 treatment protected $\beta$ cells against apoptosis through inhibition of caspase- 3 activity and attenuated cytokine-induced expression of Fas. IL-10 was previously demonstrated to suppress Fas-induced apoptosis by reducing caspase-3 activity via Fas-associated death domain-like interleukin-1-converting enzyme-like inhibitory protein upregulation and the downregulation of caspase-8 activity (49). It has been suggested that the inhibition of pro-inflammatory cytokine-mediated caspase- 3 activation by IL-10 may be crit- 
ical for pancreatic $\beta$ cell protection and survival. Therefore, the results of the present study may implicate IL-10 as a potential therapeutic agent for the treatment of T1D.

In conclusion, the present study demonstrated that IL-10 gene transfer protected against autoimmune diabetes in the pancreatic $\beta$ cells of NOD mice. The mechanism of which involved enhancing the anti-apoptotic and anti-inflammatory capacity of islets without altering the status of systemic immunity. Furthermore, these results suggested that genetic manipulation of IL-10 levels in islets may be a potential therapeutic strategy for the treatment of T1D.

\section{Acknowledgements}

The present study was supported by grants from the Natural Science Foundation of Guangdong Province (nos. S2012040006330 and S2012040006311) and the Science and Technology Program of Guangdong Province (no. 2012B031800016). The authors would like to thank Professor Zhihong Chen for technical assistance.

\section{References}

1. Bekris LM, Kavanagh TJ and Lernmark A: Targeting type 1 diabetes before and at the clinical onset of disease. Endocr Metab Immune Disord Drug Targets 6: 103-124, 2006.

2. Karlsson FA, Berne C, Björk E, et al: Beta-cell activity and destruction in type 1 diabetes. Ups J Med Sci 105: 85-95, 2000.

3. Wilson SB, Kent SC, Patton KT, et al: Extreme Th1 bias of invariant Valpha24JalphaQ T cells in type 1 diabetes. Nature 391: 177-181, 1998

4. Rabinovitch A: Immunoregulatory and cytokine imbalances in the pathogenesis of IDDM. Therapeutic intervention by immunostimulation? Diabetes 43: 613-621, 1994.

5. Tisch R and McDevitt H: Insulin-dependent diabetes mellitus. Cell 85: 291-297, 1996.

6. Delovitch TL and Singh B: The nonobese diabetic mouse as a model of autoimmune diabetes: immune dysregulation gets the NOD. Immunity 7: 727-738, 1997.

7. Zhang YC, Pileggi A, Agarwal A, et al: Adeno-associated virus-mediated IL-10 gene therapy inhibits diabetes recurrence in syngeneic islet cell transplantation of NOD mice. Diabetes 52 708-716, 2003.

8. Saraiva M and O'Garra A: The regulation of IL-10 production by immune cells. Nat Rev Immunol 10: 170-181, 2010.

9. Moore KW, de Waal Malefyt R, Coffman RL, et al: Interleukin-10 and the interleukin-10 receptor. Annu Rev Immunol 19: 683-765, 2001.

10. Ding Y, Chen D, Tarcsafalvi A, et al: Suppressor of cytokine signaling 1 inhibits IL-10-mediated immune responses. J Immunol 170: 1383-1391, 2003.

11. Nitta Y, Tashiro F, Tokui M, et al: Systemic delivery of interleukin 10 by intramuscular injection of expression plasmid DNA prevents autoimmune diabetes in nonobese diabetic mice. Hum Gene Ther 9: 1701-1707, 1998.

12. Yang Z, Chen M, Wu R, et al: Suppression of autoimmune diabetes by viral IL-10 gene transfer. J Immunol 168: 6479-6485, 2002.

13. Groux H, O'Garra A, Bigler M, et al: A CD4+ T-cell subset inhibits antigen-specific T-cell responses and prevents colitis Nature 389: 737-742, 1997.

14. Asseman C, Mauze S, Leach MW, Coffman RL and Powrie F: An essential role for interleukin 10 in the function of regulatory $\mathrm{T}$ cells that inhibit intestinal inflammation. J Exp Med 190 995-1004, 1999.

15. Levings MK, Sangregorio R, Galbiati F, et al: IFN-alpha and IL-10 induce the differentiation of human type $1 \mathrm{~T}$ regulatory cells. J Immunol 166: 5530-5539, 2001.

16. Goudy KS, Burkhardt BR, Wasserfall C, et al: Systemic overexpression of IL-10 induces CD4+CD25+cell populations in vivo and ameliorates type 1 diabetes in nonobese diabetic mice in a dose-dependent fashion. J Immunol 171: 2270-2278, 2003.
17. Xu AJ, Chen ZH, Tian F, Yan LH and Li T: Effects of adenovirus-mediated interleukin-10 gene transfer on apoptosis and insulin secretion function of beta cell. Chinese Medical Journal 90: 1711-1715, 2010 (In Chinese).

18. Xu AJ, Zhu W, Tian F, Yan LH and Li T: Recombinant adenoviral expression of IL-10 protects beta cell from impairment induced by pro-inflammatory cytokine. Mol Cell Biochem 344: 163-171, 2010.

19. Marselli L, Dotta F, Piro S, et al: Th2 cytokines have a partial, direct protective effect on the function and survival of isolated human islets exposed to combined proinflammatory and Th1 cytokines. J Clin Endocrinol Metab 86: 4974-4978, 2001.

20. Casellas A, Salavert A, Agudo J, et al: Expression of IGF-I in pancreatic islets prevents lymphocytic infiltration and protects mice from type 1 diabetes. Diabetes 55: 3246-3255, 2006.

21. van Belle TL, Coppieters KT and von Herrath MG: Type 1 diabetes: Etiology, immunology, and therapeutic strategies. Physiol Rev 91: 79-118, 2011.

22. Thomas HE, Graham KL, Chee J, et al: Proinflammatory cytokines contribute to development and function of regulatory $\mathrm{T}$ cells in type 1 diabetes. Ann NY Acad Sci 1283: 81-86, 2013.

23. Serreze DV, Chapman HD, Post CM, et al: Th1 to Th2 cytokine shifts in nonobese diabetic mice: sometimes an outcome, rather than the cause, of diabetes resistance elicited by immunostimulation. J Immunol 166: 1352-1359, 2001.

24. Asadullah K, Sterry W and Volk HD.Interleukin-10 therapy - review of a new approach. Pharmacol Rev 55: 241-269, 2003.

25. Pennline KJ, Roque-Gaffney E and Monahan M: Recombinant human IL-10 prevents the onset of diabetes in the nonobese diabetic mouse. Clin Immunol Immunopathol 71: 169-175, 1994.

26. Goudy K, Song S, Wasserfall C, et al: Adeno-associated virus vector-mediated IL-10 gene delivery prevents type 1 diabetes in NOD mice. Proc Natl Acad Sci USA 98: 13913-13918, 2001.

27. Suk K, Kim S, Kim YH, et al: IFN-gamma/TNF-alpha synergism as the final effector in autoimmune diabetes: a key role for STAT1/IFN regulatory factor-1 pathway in pancreatic beta cell death. J Immunol 166: 4481-4489, 2001.

28. Tang Q, Henriksen KJ, Boden EK, et al: Cutting edge: CD28 controls peripheral homeostasis of CD4+CD25+ regulatory T cells. J Immunol 171: 3348-3352, 2003.

29. Chen Z, Herman AE, Matos M, Mathis D and Benoist C: Where CD4+CD25+ Treg cells impinge on autoimmune diabetes. J Exp Med 202: 1387-1397, 2005.

30. Feuerer M, Shen Y, Littman DR, Benoist C and Mathis D: How punctual ablation of regulatory $\mathrm{T}$ cells unleashes an autoimmune lesion within the pancreatic islets. Immunity 31: 654-664, 2009.

31. Herman AE, Freeman GJ, Mathis D and Benoist C: CD4+CD25+ $\mathrm{T}$ regulatory cells dependent on ICOS promote regulation of effector cells in the prediabetic lesion. J Exp Med 199: 1479-1489, 2004.

32. Gregori S, Giarratana N, Smiroldo S and Adorini L: Dynamics of pathogenic and suppressor $\mathrm{T}$ cells in autoimmune diabetes development. J Immunol 171: 4040-4047, 2003.

33. Salomon B, Lenschow DJ, Rhee L, et al: B7/CD28 costimulation is essential for the homeostasis of the CD4+CD25+ immunoregulatory $\mathrm{T}$ cells that control autoimmune diabetes. Immunity 12 : 431-440, 2000.

34. Walker LS: Natural Treg in autoimmune diabetes: all present and correct? Expert Opin Biol Ther 8: 1691-1703, 2008.

35. Szanya V, Ermann J, Taylor C, Holness C and Fathman CG: The subpopulation of CD4+CD25+ splenocytes that delays adoptive transfer of diabetes expresses L-selectin and high levels of CCR7. J Immunol 169: 2461-2465, 2002.

36. Kay TW, Thomas HE, Harrison LC and Allison J: The beta cell in autoimmune diabetes: many mechanisms and pathways of loss. Trends Endocrinol Metab 11: 11-15, 2000

37. Cnop M, Welsh N, Jonas JC, et al: Mechanisms of pancreatic beta-cell death in type 1 and type 2 diabetes: many differences, few similarities. Diabetes 54 (Suppl 2): S97-S107, 2005.

38. Katz JD, Benoist C and Mathis D: T helper cell subsets in insulin-dependent diabetes. Science 268: 185-1188, 1995.

39. Kurrer MO, Pakala SV, Hanson HL and Katz JD: Beta cell apoptosis in T cell-mediated autoimmune diabetes. Proc Natl Acad Sci USA 94: 213-218, 1997.

40. Kim YH, Kim S, Kim KA, et al: Apoptosis of pancreatic beta-cells detected in accelerated diabetes of NOD mice: no role of Fas-Fas ligand interaction in autoimmune diabetes. Eur J Immunol 29: 455-465, 1999. 
41. Eizirik DL and Mandrup-Poulsen T: A choice of death - the signal-transduction of immune-mediated beta-cell apoptosis. Diabetologia 44: 2115-2133, 2001.

42. Slee EA, Adrain C and Martin SJ: Serial killers: ordering caspase activation events in apoptosis. Cell Death Differ 6: 1067-1074, 1999

43. Wolf BB, Schuler M, Echeverri F and Green DR: Caspase-3 is the primary activator of apoptotic DNA fragmentation via DNA fragmentation factor-45/inhibitor of caspase-activated DNase inactivation. J Biol Chem 274: 30651-30656, 1999.

44. Loweth AC, Williams GT, James RF, Scarpello JH and Morgan NG: Human islets of Langerhans express Fas ligand and undergo apoptosis in response to interleukin-lbeta and Fas ligation. Diabetes 47: 727-732, 1998 .

45. Riachy R, Vandewalle B, Moerman E, et al: 1,25-Dihydroxyvitamin D3 protects human pancreatic islets against cytokine-induced apoptosis via down-regulation of the Fas receptor. Apoptosis 11: 151-159, 2006.
46. Zhou JH, Broussard SR, Strle K, et al: IL-10 inhibits apoptosis of promyeloid cells by activating insulin receptor substrate-2 and phosphatidylinositol 3'-kinase. J Immunol 167: 4436-4442, 2001.

47. Dhingra S, Sharma AK, Arora RC, Slezak J and Singal PK: IL-10 attenuates TNF-alpha-induced NF kappaB pathway activation and cardiomyocyte apoptosis. Cardiovasc Res 82: 59-66, 2009.

48. Londoño D, Carvajal J, Strle K, Kim KS and Cadavid D: IL-10 Prevents apoptosis of brain endothelium during bacteremia. J Immunol 186: 7176-7186, 2011.

49. Bharhani MS, Borojevic R, Basak S, et al: IL-10 protects mouse intestinal epithelial cells from Fas-induced apoptosis via modulating Fas expression and altering caspase- 8 and FLIP expression. Am J Physiol Gastrointest Liver Physiol 291: G820-G829, 2006. 